\title{
Deep Brain Stimulation in Neurosurgical Practice and Research: Its Application and Ethical Issues
}

\begin{abstract}
Nurunanbi ASM ${ }^{1}$, Mozaffor $\mathbf{M}^{2}$, Islam MJ $^{3}$, Haroon $\mathrm{K}^{4}$
Conflict of interest: There is no conflict of interest relevant to this paper to disclose.

Funding agency: This project was not funded by any group, organization or institution.

Contribution of the authors: ASM Nurunnabi and M Mozaffor were involved in concept and design of the review paper; ASM Nurunnabi, M Mozaffor, MJ Islam and $\mathrm{K}$ Haroon were equally involved in the literature search, review, compilation, manuscript writing and revision.

Data collection-

Abstract:

Deep brain stimulation (DBS), a form of neuromodulation, is recognized as a therapeutic venture for the management of chronic pain, the evaluation and management of epilepsy, and the treatment of Parkinson's disease and other movement disorders. Clinical investigators are conducting more trials for its further use in obsessive compulsive disorders (OCD), depression, and traumatic brain injury. It is imperative to address the ethical concerns of such innovative neurosurgical treatment and research with human experimentation in the respective arena. The term 'neurosurgical ethics' is a moral approach towards ethical problems emerging from our day to day neurosurgical practice and research as well as current and future innovation in the field. The review paper outlined the procedures of DBS, as currently used in therapeutic and experimental applications and to discuss the ethical concerns regarding this innovative procedure.
\end{abstract}

Scalp block with anaesthesia-

Editorial formatting-

Copyright: @2020bang.BJNS published by BSNS. This article is published under the creative commons CC-BY-NC license. This license permits use distribution (https://creativecommons. orgf/licences/by-nc/4-0/)reproduction in any medium, provided the original work is properly cited and is not used for commercial purposes.

Received: 05.01 .20

Accepted: 08.03 .20

\section{Introduction:}

Neurosurgery is among the newest of all surgical disciplines, appearing at the dawn of the twentieth century ${ }^{1}$. Neurosurgeons have the training and education to operate on the organ that constitutes the locus of consciousness, body functions, emotions, and intelligence of human beings ${ }^{2}$. The ethical dilemmas are inherent while acting upon and manipulating the brain and nerves and are

Key words: Deep brain stimulation, innovative neurosurgery, neurosurgical treatment, neurosurgical research, ethical issues, neurosurgical ethics.

Bang. J Neurosurgery 2020; 10(1): 92-96

compounded by the more or less same moral questions which are common to all medical fields ${ }^{1,2}$. The term 'neurosurgical ethics' is a moral approach towards ethical problems emerging from our day to day neurosurgical practice and research as well as current and future innovation in the field ${ }^{1-3}$. Deep brain stimulation (DBS), a form of neuromodulation, is recognized as an innovative therapeutic venture in today's neurosurgery 3,4 . Currently it is used for the

1. Dr. Abu Sadat Mohammad Nurunanbi, Assistant Professor, Department of Anatomy, OSD, Directorate General of Health Services (DGHS), Dhaka, Bangladesh.

2. Dr. Miliva Mozaffor, Clinician Researcher, Biomedical Research Foundation (BRF), Dhaka, and Lecturer, Department of Biochemistry, Medical College for Women \& Hospital, Uttara, Dhaka, Bangladesh.

3. Dr. Md. Joynul Islam, Associate Professor, Department of Clinical Neurosurgery, National Institute of Neurosciences \& Hospital, Dhaka, Bangladesh.

4. Dr. Kaisar Haroon, Assistant Professor, Department of Clinical Neurosurgery, National Institute of Neurosciences \& Hospital, Dhaka, Bangladesh.

Address for Correspondence: Dr. Abu Sadat Mohammad Nurunanbi, Assistant Professor, Department of Anatomy, OSD, Directorate General of Health Services (DGHS), Dhaka, Bangladesh. Email: shekhor19@yahoo.com ORCiD: 0000-0002-5455-7838 
management of chronic pain, the evaluation and management of epilepsy, and the treatment of Parkinson's disease and other movement related disorders like essential tremor and dystonia ${ }^{4-6}$. The US Food and Drug Administration (FDA) approved the use of the deep brain stimulator for refractory Parkinson's disease in $2002^{4}$, while clinical investigators are conducting more and more trials for its further use in obsessive compulsive disorders (OCD), depression, and traumatic brain injury ${ }^{4-6}$. However, the "boundary between experimental work and therapeutic deployment" lies not only on approval of the regulatory authorities like $\mathrm{FDA}^{3}$, but also on the consensus of the medical community that the putative treatment is effective and safe $\mathrm{se}^{2,3,4}$, and that the intended benefits will appear without any short or long-term adverse effects $2,3,7,8$. In recent years, neurosurgical ethics has become more relevance as changes in society and technology have brought some inevitable questions into focus ${ }^{4}$. The former changes as evident in movements toward patient-centered surgery, evidence-based medicine, and cost-effective care, which resulting in reconsidering the relationship between innovative neurosurgery and our societal aims ${ }^{4,9}$. The latter changes observed by a tremendous expansion of the horizons of innovation for interfacing with the human brain and spine that consequentially demand us to use our philosophical reasoning to assess the merits of those technical innovations ${ }^{1,3,4}$. Hence, the present review paper aims to outline the procedures, progress and prospects of DBS as used in therapeutic and experimental applications and simultaneously discuss the ethical concerns regarding this innovative procedure.

\section{Methods:}

This review paper is based on an extensive but relevant literature search in HINARI, Pubmed and Google Scholar databases done in between August and December of 2019. Key words used for searching were: deep brain stimulation, innovative neurosurgery, neurosurgical treatment, neurosurgical research, ethical issues and neurosurgical ethics. Literatures were sourced from a range of neurosurgical, neurological, and psychiatric as well as bioengineering, social studies, bioethics and philosophical publications. Review of literature included both the original research and review articles and some western professional association's guidelines.

\section{Results:}

After meticulous scrutiny and review, we identified 20 published articles and 2 institutional/professional guidelines most relevant to our topic. We tried to focus on history, progress, current uses and future prospects of DBS as well as related ethical issues of adopting DBS in practice and research in the field of neurosurgery. Major ethical concerns are selection of patients for the treatment or research, informed consent, justifiability of clinical trial design, weighing benefits against risks, confidentiality of health data and data sharing, DBS in the paediatric population, potential use of DBS for brain enhancement, any direct modification of behaviour through neuromodulation, and last but not the least, resource allocation in such costly healthcare practice.

\section{Discussion:}

Deep brain stimulation - History, procedure, prospects

Deep brain stimulation (DBS) originates from the developments in stimulation of the cerebral cortex of animals and cortical functional localization, which took place in the late 19th century $4,7,10-13$. Since then stereotactic frame was developed which facilitated stimulation of deeper brain regions at experimental level. Later in 1947, introduction of X-ray pneumoencephalography greatly improved surgeons' ability to localise the target zone $\mathrm{e}^{7,10-13}$. In 1950s, through lesioning to treat tremor, modern stereotactic techniques were used "to ablate the ventrolateral or ventroanterior nuclei of the thalamus, along with intraoperative electrical stimulation and recording being employed to localise targets" ${ }^{\prime 10}$. In 1960s, further improvement was done by a group of scientists from France, as they reported substantial alleviation of tremor in Parkinsonism through "high-frequency (approx. 100-200 Hz) electrical stimulation of the ventral intermediate thalamic nucleus" 10 . Consequentially, more advancement was in place through implantable spinal cord stimulators, which paved the path to introduction of implantable DBS device, targeted on nonhuman primates ${ }^{10,11,13}$. The success ultimately translated into clinical practice, as researchers could use DBS in sub-thalamic nucleus region effectively in the treatment of motor symptoms in Parkinson's disease ${ }^{10-13}$. Similarly, the internal globus pallidus was also identified as a viable target for DBS ${ }^{10,12,13}$. By the end of the decade, clinical use of DBS became widespread in the treatment of Parkinsonian disorders $4,7,10-14$. In 2009, 
FDA approved DBS as a 'Humanitarian Device Exemption' for the treatment of chronic, severe, and treatment-resistant obsessive compulsive disorder (OCD), which was the beginning of an era in neuropsychiatric treatment and research ${ }^{14}$.

Deep brain stimulation (DBS), a form of neuromodulation, is a functional neurosurgical approach in which a high-frequency electrical current stimulates targeted brain structures for therapeutic benefit ${ }^{7,8,14}$. The system include an implanted brain electrode (into deep brain regions) by using stereotactic techniques and a pacemaker-like implanted pulse generator (IPG), which is typically placed subdermally below the clavicle $7,10,14$. A modern IPG contains a battery along with electronic components that deliver electrical stimulation and can be externally controlled (such as frequency, pulse width, and voltage) by patients or clinicians ${ }^{10}$. Although physiological mechanism of DBS is not fully understood, it is generally assumed that it works through excitation and/or inhibition of neuronal cell bodies adjacent to the electrode and on nearby axons, too $10,12,14,15$. This intervention is based on the empirical observation that specific brain targets stimulated by electro-magnetic wave tends to mimic the effect of a lesion without the need for destroying brain tissue $8,14,15$. Low-frequency stimulation appears to be effective, while high-frequency stimulation may reduce activity and induce functional lesion; however, such simplistic view is controversial to date ${ }^{10,13,15}$.

Parkinson's disease, essential tremor and dystonia are the most widely accepted indications for DBS. DBS also shows promise in chronic pain, obsessive compulsive disorder (OCD), epilepsy, and depression ${ }^{8-}$ 10,14,16,17. Moreover, addiction, anorexia, obesity, Tourette's, Alzheimer's, enhancement of memory in various neurological disorders including neurologic diseases, traumatic brain injury and other injuries such as stroke, cardiac arrest, or encephalitis as well as increasing learning capacity in individuals are all in the list of future possibilities and in some cases, limited trials are being undertaken ${ }^{10,12-17}$. The future of DBS depends on technological advances in the area: the focus should be the improvement of clinical knowledge as well as improved practicality e.g. smaller size devices, increased battery life, greater tolerance and safety profiles, and improved software ${ }^{12,13}$.

\section{Ethical issues in deep brain stimulation}

The traditional practice of neurosurgery have some ethical and legal implications, for example, issues related to withdrawal of life-sustaining treatment in irreversible head injury leading to brain death or persistent vegetative state; or decision-making for such patients (for incapacitated patients); or removal of ventilator from a quadriplegic patient with cervical spinal cord injury where recovery is unlikely $3,6,8,14,18$. Those issues have already been debated, discussed, and written about during the latter part of the 20th century in the literatures of philosophy or medical ethics, decisions imposed by the jurisdictions or judicial authority or codes of institutional ethics committees ${ }^{1-}$ $3,8-10,14,18$. Neurosurgery is inherently a risky job; in western countries, neurosurgeons are frequently the subject of medical-legal action either to defend medical malpractice or to give expert opinion in the court $6,8,14$. Several neurosurgical societies have crafted codes of ethics as guidelines for their members who testify in medical malpractice cases ${ }^{19,20}$.

Like any other fields, future advances in technology and disease understanding will likely lead to unforeseen treatments in the field of neurosurgery, too. Hence, the innovative field of DBS practice and research focuses on multitude of neuroethical issues, such as patient selection criteria; informed consent for treatment and research, especially incapacitated patients and paediatric patients for any diagnostic and prognostic purposes; the subjectivity of data interpretation and confidentiality of health data and data sharing as well ${ }^{3,6,8,10,13,14,18,21-22}$. Especially, a careful informed consent process should be in place in which unrealistic expectations of patients and their families are addressed and in which special attention is given to competence of the treatment or research team $^{6,8,14}$. In the above mentioned situations, existing laws in the territory, institutional guidelines or professional code of ethics may clarify what are the do's and don'ts ${ }^{14,18,19,20 .}$

On weighing benefits against risks, the clinical application of deep brain stimulation proceeded for years without clear understandings of its mechanisms of action or extensive preclinical studies of safety and efficacy $8,10,13,15$. Further understanding of the mechanisms underlying cerebral circuits in epilepsy and its comorbidities is required, in order to further define specific criteria and predictors in the selection of patients who could benefit from $\mathrm{DBS}^{8,10}$. Although DBS is considered a relatively safe neurosurgical 
procedure with low mortality, some intraoperative, immediate and late postoperative complications have occurred like intracranial bleeding, seizure, stroke, hardware malfunction, infection, prolonged length of hospitalization, and postoperative confusion ${ }^{8,23}$. Recent evidence suggests loss of neurons proximal to the active electrode, while the resulting lesions were not adequate to treat the disorder and efficacy is doubtful and sometimes required continued stimulation over a longer period of time $8,10,23$. Moreover, the complication rate was found $>25 \%$, and permanent neurologic damage was $4-6 \%^{8}$. Hence, it is still critical to understand the origin of adverse events and to ensure the non damaging stimulation in situ, which is of deep ethical concern. It was found that quality of life on aspects such as emotional well-being, social support, and interpersonal relationships may actually decrease after surgery, even when physical symptoms improve $^{5,10,14,18,20,24-26}$. Therefore, DBS should be used only when a patient's condition is refractory to all other interventions and when there is a high probability of improvement of patients' quality of life $^{8,10,14,18,21-23}$.

In DBS, successfully translating research into clinical use is very much impeded by difficulty in designing suitable clinical trials, blinding (as patients are aware of the stimulation being on or off), chances of falsenegative results (due to stimulation below the threshold), overwhelmed clinical efficacy and publishing that results $6,8,10,18,24,25$. DBS has received a large amount of publications and press in recent years, often describing the treatment a miraculous cure to the disease and disabilities. Hence, any failure of these expectations can be a psychological damage to the patients. Researchers need to be careful and avoid overselling the positive effects $8,24,25$. Similar concerns prevail in enhancement of memory and learning, which is a future prospect of DBS in patients with severely compromised memory due to different conditions. There is a common fear that memorymodulating technologies might be used to fundamentally change people's self-identity, self-image and manipulations with their memories may happen in some events ${ }^{6,8,14,24}$. An important step to further development of responsible and transparent practice and research in the field would be the establishment of a comprehensive registry system and a national guideline in the country $6,8,10,14$.
Last but not the least, financial cost is a remarkable impediment to DBS treatment and research and its widespread clinical adoption. The device itself, and the surgery and follow-up as well as large-scale trials seem very hard to secure funding, even in developed countries $8,10,14,18,24$. It is more problematic in the developing countries like Bangladesh, where the settings are resource-poor and very few people can afford the hardware and treatment costs. This is more likely to generate disparities in access to treatment, implying inequalities between patients and significant economic, family, and social challenges ${ }^{14,18,24}$, even when there is a high prevalence of movement disorders and other conditions in the population that may be responsive to DBS treatment.

\section{Conclusion:}

Deep brain stimulation is an established treatment in neurology and is emerging as experimental treatment in the field of neurosurgery. Over the past years, bioethicists and philosophers have been working in close cooperation with clinicians and researchers to identify and discuss the most important ethical issues in both clinical practice and research. There is a growing corpus of literature available that addresses these issues. It is expected that this review paper will be a provocation to the physicians, administrators, researchers and policy makers to dig into the issues.

\section{References:}

1. Ammar A, Bernstein M. eds. Neurosurgical ethics in practice: value-based medicine. Berlin: Springer-Verlag; 2014. p.8-10.

2. Awad IA. ed. Philosophy of neurological surgery. New York: Thieme Medical Publishers; 1995. p.19-22.

3. Fins JS. Deep Brain Stimulation: Ethical Issues in Clinical Practice and Neurosurgical Research. In: Krames ES, Peckham PH, Rezai AR. eds. Neuromodulation. London: Elsevier; 2009. p.81-91.

4. Gardner J. A history of deep brain stimulation: Technological innovation and the role of clinical assessment tools. Soc Stud Sci. 2013;43(5):707-28.

5. Raihan MZ, Aziz T. Deep brain stimulation in sub-thalamic nucleus in idiopathic Parkinson's disease - our initial experience in four cases. Bang $J$ Neurosurgery. 2019;9(1):78-83.

6. Schermer M. Ethical issues in deep brain stimulation. Front Integr Neurosci. 2011;5:e17.

7. Coffey RJ. Deep brain stimulation devices: a brief technical history and review. Artif Organs. 2009;33(3):208-20.

8. Clausen J. Ethical brain stimulation - neuroethics of deep brain stimulation in research and clinical practice. Eur $\mathrm{J}$ Neurosci. 2010;32:1152-62. 
9. Grill WM. Safety considerations for deep brain stimulation: review and analysis. Expert Rev Med Devices. 2005;2(4):409-20.

10. Pycroft L, Stein J, Aziz T. Deep brain stimulation: an overview of history, methods, and future developments. Brain Neurosci Adv. 2018;2:1-6.

11. Schwalb JM, Hamani C. The history and future of deep brain stimulation. Neurotherapeutics. 2008;5(1):3-13.

12. Miocinovic S, Somayajula S, Chitnis S, Vitek JL. History, applications, and mechanisms of deep brain stimulation. JAMA Neurol. 2013;70(2):163-71.

13. Zangiabadi N, Ladino LD, Sina F, Orozco-Hernández JP, Carter A, Téllez-Zenteno JF. Deep Brain Stimulation and Drug-Resistant Epilepsy: A Review of the Literature. Front Neurol. 2019;10:601.

14. Johansson V, Garwicz M, Kanje M, Halldenius L, Schouenborg J. Thinking ahead on deep brain stimulation: an analysis of the ethical implications of a developing technology. AJOB Neuroscience. 2014;5(1):24-33.

15. Herrington TM, Cheng JJ, Eskandar EN. Mechanisms of deep brain stimulation. J Neurophysiol. 2016;115(1): 19-38.

16. Kocabicak E, Temel Y, Höllig A. Current perspectives on deep brain stimulation for severe neurological and psychiatric disorders. Neuropsychiatr Dis Treat. 2015;11:1051-66.

17. Halpern C, Hurtig H, Jaggi J, Grossman M, Won M, Baltuch G. Deep brain stimulation in neurologic disorders. Parkinsonism Relat Disord. 2007;13(1):1-16.
18. Unterrainer M, Oduncu FS. The ethics of deep brain stimulation (DBS). Med Health Care Philos. 2015;18(4):47585.

19. World Federation of Neurosurgical Societies. Committee for Ethics and Medico-Legal Affairs. Statement of Ethics in Neurosurgery. Retrieved from: https://www.wfns.org/ W F N S D a $t$ a / D o c u m e $n t /$ Statement_of_Ethics_in_Neurosurgery.pdf. (Accessed August 04, 2019).

20. American Association of Neurological Surgeons (AANS). The Code of Ethics of the American Association of Neurological Surgeons. Retrieved from: https:// www.aans.org/-/media/Images/AANS/Header/ Govenance/AANS_Code_of_Ethics_11-22-2014. (Accessed August 04, 2019).

21. Glannon W. Consent to deep brain stimulation for neurological and psychiatric disorders. J Clin Ethics. 2010;21(2):104-11.

22. Copeland SM. Problems with seeing DBS recipients primarily as research subjects. AJOB Neuroscience. 2013;4(2):50-2.

23. Fang JY, Tolleson C. The role of deep brain stimulation in Parkinson's disease: an overview and update on new developments. Neuropsychiatr Dis Treat. 2017;13: 723-32.

24. Bell E, Mathieu G, Racine E. Preparing the ethical future of deep brain stimulation. Surg Neurol. 2009;72(6):577-86.

25. Schlaepfer TE, Fins JJ. Deep brain stimulation and the ethics of responsible publishing. JAMA. 2010;303(8):775-6. 